\title{
A!
}

This is an electronic reprint of the original article.

This reprint may differ from the original in pagination and typographic detail.

Latifi, Kourosh; Wijaya, Harri; Zhou, Quan

\section{Multi-particle acoustic manipulation on a Chladni plate}

\section{Published in:}

Proceedings of the IEEE International Conference on Manipulation, Automation and Robotics at Small Scales, MARSS 2017

DOI:

10.1109/MARSS.2017.8001920

Published: 17/07/2017

Document Version

Peer reviewed version

Please cite the original version:

Latifi, K., Wijaya, H., \& Zhou, Q. (2017). Multi-particle acoustic manipulation on a Chladni plate. In Proceedings of the IEEE International Conference on Manipulation, Automation and Robotics at Small Scales, MARSS 2017 (pp. 1-7). IEEE. https://doi.org/10.1109/MARSS.2017.8001920

This material is protected by copyright and other intellectual property rights, and duplication or sale of all or part of any of the repository collections is not permitted, except that material may be duplicated by you for your research use or educational purposes in electronic or print form. You must obtain permission for any other use. Electronic or print copies may not be offered, whether for sale or otherwise to anyone who is not an authorised user. 


\title{
Multi-particle acoustic manipulation on a Chladni plate
}

\author{
Kourosh Latifi, Harri Wijaya, and Quan Zhou \\ Department of Electrical Engineering and Automation, \\ School of Electrical Engineering, Aalto University \\ Espoo, Finland \\ kourosh.latifi@aalto.fi; harri.wijaya@aalto.fi; quan.zhou@aalto.fi
}

\begin{abstract}
Controlling the motion of multiple miniature objects independently and simultaneously is a grand challenge in microrobotics. In this paper, we report our recent achievements in acoustic manipulation that can control the motion of multiple objects simultaneously and independently on a centrally actuated vibrating plate. By employing spatially highly nonlinear excitation fields on the plate, we can control the motion of multiple objects with relaxed properties. The paper reports the modelling of the excitation fields, open-loop control based on pre-calculated control sequence, and closed loop control using model predictive control (MPC) and linear programming methods. The experimental results show that with appropriate planning, object motion on vibrating plate is sufficiently predictable to be controlled even in open-loop. The experimental results with closed-loop control show that the methods allow various applications including trajectory following, particle assembling, and droplet merging. Despite the reported method is based on acoustic manipulation on a Chladni plate, the method can be extended to other energy fields as soon as they are spatially highly nonlinear and such nonlinearity can be excited.
\end{abstract}

Keywords-accoustic manipulation; Chladni plate; mobile microrobot; model predictive control; linear programming.

\section{INTRODUCTION}

Controlling the motion of miniature objects on solid surfaces or in fluids has numerous applications from manipulation of microparticles, cells and other organisms on a microfluidic chip [1]-[6], to microassembly inside liquid [7] and moving droplets on biochips [8], [9]. A great variety of systems have been developed, by applying different energy fields or harvesting local energy or power sources, e.g. electrostatic force [10], [11], electromagnetic [12], [13], acoustic [4], [6], optical [14], thermal [15], chemical [16], and bacteria propelled [17] systems. The miniature objects controlled by those manipulation systems are often called mobile microrobots or mobile micro agents. Often, such systems can control the motion of single objects or a group of objects where the motion of the objects is similar.

Despite the remarkable achievements in controlling the motion of miniature objects, it remains a grand challenge to control the motion of multiple objects independently and simultaneously. To date, only limited successes have been achieved in independent motion control of multiple objects, mostly by designing miniature objects with distinct properties. For example, individual objects are designed with different mechanical properties so that they react distinctly to a uniform excitation signal in electrostatic manipulation [10]. Two microrobots are designed to possess different mechanical resonant frequencies allowing independent motion control in magnetic manipulation [12], where motion of microrobots can be switched on or off by varying the frequency of the magnetic field. The geometry of microrobots are designed to respond uniquely to the same applied magnetic fields allowing simultaneous manipulation in a dissimilar fashion [18], [19]. All those work requires careful design or selection of the properties of individual micro objects, which greatly limits their potential applications.

This paper attacks the problem from a different angle, where we use a spatially highly nonlinear excitation field for independent and simultaneous acoustic manipulation of multiple objects. We apply an extremely simple physical setup - a centrally-actuated vibrating plate, known as Chladni plate, where just a single actuator mounted at the center of the plate is used to drive the system. By changing the driving frequency, the plate offers complex frequency-dependent two-dimensional excitation fields on the plate surface. Those fields allow us to control the motion of multiple objects independently and simultaneously for a wide range of objects where the properties of individual objects may or may not be the same. Despite its remarkable ability, our hardware is the simplest among all acoustic manipulation systems [1], [4], [20]-[23].

In this paper, we summarize our recent works on acoustic manipulation of multiple particles on a Chladni plate. We discuss the modelling method for such spatially nonlinear and frequency-dependent fields, and several open-loop and closedloop control methods implemented for the system. Then, we experimentally study the capabilities of different control methods and report their achievements. This work is based on our recent paper [24], with broader perspective and additional analysis and experimental results.

\section{EXPERIMENTAL SETUP}

The experimental platform is shown in Fig. 1. The setup consists of a diced silicon plate $(50 \mathrm{~mm} \times 50 \mathrm{~mm} \times 525 \mu \mathrm{m})$, actuated by a centrally-mounted piezoelectric actuator (Piezomechanik/PSt 150/2x3/5). The piezoelectric actuator is mounted to the central backside of the plate using cyanoacrylate adhesive. To excite the plate, the actuation signal is generated within a control PC, sent out through a D/A converter (National Instruments/USB-6363), and amplified (Piezosystems/EPA104-230) to the piezoelectric actuator. The particles to be manipulated are placed on the vibrating plate. The plate is imaged by a camera (ImperX/IGV-B1621C-KC000 with 


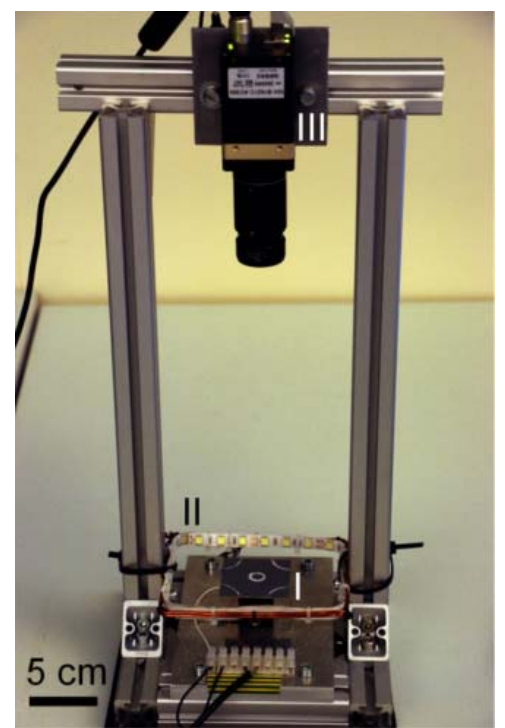

Fig. 1. Experimental setup. I: silicon plate, II: ring of LEDs, III: camera.

Infinity/InfiniMite Alpha lens) during particle manipulation. The camera is connected to the control PC to feedback the positions of particles. To provide better contrast, a ring of LEDs was mounted horizontally around and slightly above the plate.

\section{Modelling Of THE Motion ON ChLADNi Plate}

Extensive effort has been devoted to model Chladni patterns and resulted particle motion using PDE eigenvalue solutions [25]. However, a centrally-actuated Chladni plate should be considered as a driven oscillation system where resonance frequencies are different from Eigen frequencies. In a recent work [26], theoretical model was put forward for a centrallyactuated Chladni plate based on solving the two-dimensional inhomogeneous Helmholtz equation. However, the mentioned theoretical model does not carefully explain the particle motion on the plate except in certain frequencies.

We developed a fully data-driven method to model the particle motion using particle tracking velocimetry (PTV), as illustrated in Fig. 2. We evenly placed numerous (mostly greater than 100) compressed $600 \mu \mathrm{m}$ solder balls over the plate. Then, we excited the plate with notes where each note was attributed by a certain frequency, voltage amplitude, and duration. The selection of frequency of each note can be based on different criteria, e.g. the frequency with most significant particle motion. In [24], we selected the notes according to western musical theory, from the chromatic scale from C6 $(1.047 \mathrm{kHz})$ up to A\#10 (29.83 kHz). Voltage amplitudes vary with frequencies, and have been selected in the range of 1.49 volts up to 90 volts. To create the model of particle motion, we measured the position $p \in R^{2}$ of each particle before and after every excitation using the top-view camera, and calculated the displacement $\Delta p \in R^{2}$. For all different notes, a dataset $(p, \Delta p)$ were created from the measurement data. We used locally weighted scatterplot smoothing (LOESS) regression method to fit a model to the data points as

$$
\Delta p=u_{n}(p)+e
$$

a

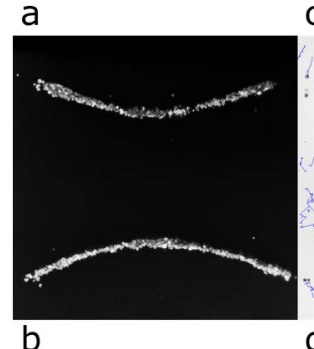

C

e

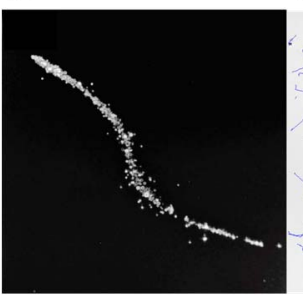

d

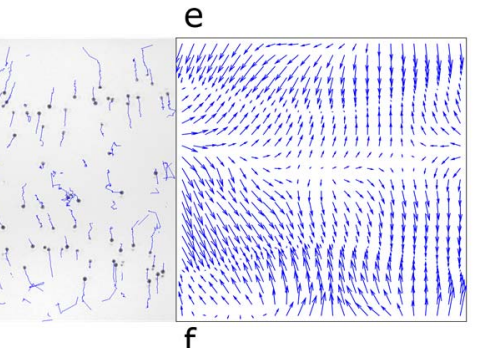

f

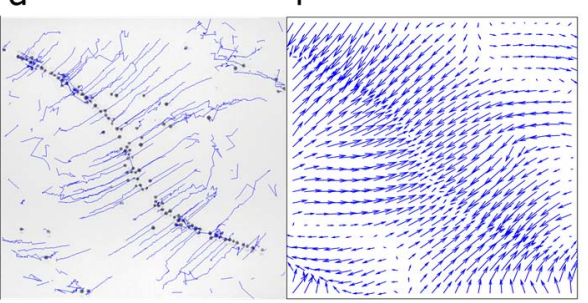

Fig. 2. (a-b) Chladni figures visualized with salt particles, (c-d) resulted PTV, and (e-f) modelled displacement fields for excitation frequencies of $1868 \mathrm{~Hz}$ and $2975 \mathrm{~Hz}$, respectively.

Where $u_{n}$ is a two-dimensional displacement model fitted for note $\mathrm{n}, n \in 1 \ldots N, N$ the number of notes, and $e$ is the model residual. After fitting $u_{n}$, we computed $|e|^{2}=\left|\Delta p-u_{n}(p)\right|^{2}$ for each data point, and used a similar fitting procedure for $(p$, $|e|^{2}$ ) dataset to find the residual field of each note as a function of position $\left(\xi_{n}{ }^{2}: R^{2} \rightarrow R\right)$.

Details of the modelling procedure using the western musical note are explained in [24]. Fig. 3 illustrates the theoretically computed Chladni figures, modelled vector fields, and residual fields. The experimentally obtained displacement fields can be related to theoretically predicted Chladni figures, but with significant difference.

\section{CONTROL STRATEGY}

\section{A. Open-loop control strategy}

A simulation framework is constructed based on the displacement and residual models. We use the simulation framework to generate the required sequence of notes for a predefined manipulation task. The generated sequence is then replayed in real setup to control the particle motion in open-loop. We use two methods to generate the notes sequence for openloop control experiments, direct method and Neighborhood Net Motion method.

\section{1) Direct method}

In this method, the notes sequence is generated by simulating the motion of one particle towards its target. At a given point, the note to be played is selected based on the control algorithm explained in [24]. In order to include the motion uncertainty, we use the noise factor calculated from the residual field model. The position of particle is then updated by adding the displacement vector and the noise factor of the current position. The procedure is repeated until the target is reached. The note in each iteration is recorded to be replayed in the real setup.

\section{2) Neighborhood Net Motion method}

The system behavior of Chladni plate is chaotic and the object motion is sensitive to its initial position. Even $1 \mathrm{~nm}$ 
change in initial position can lead up to $100 \mu \mathrm{m}$ difference in the trajectories [24]. In experiment, such initial position error is unavoidable. The accuracy of the vision system could easily contribute to such positioning error.
In order to incorporate such initial position error in openloop planning, for a given initial position we shall select the note such that the displacement field will move not only the particle at the given position but also when the particle is slightly off
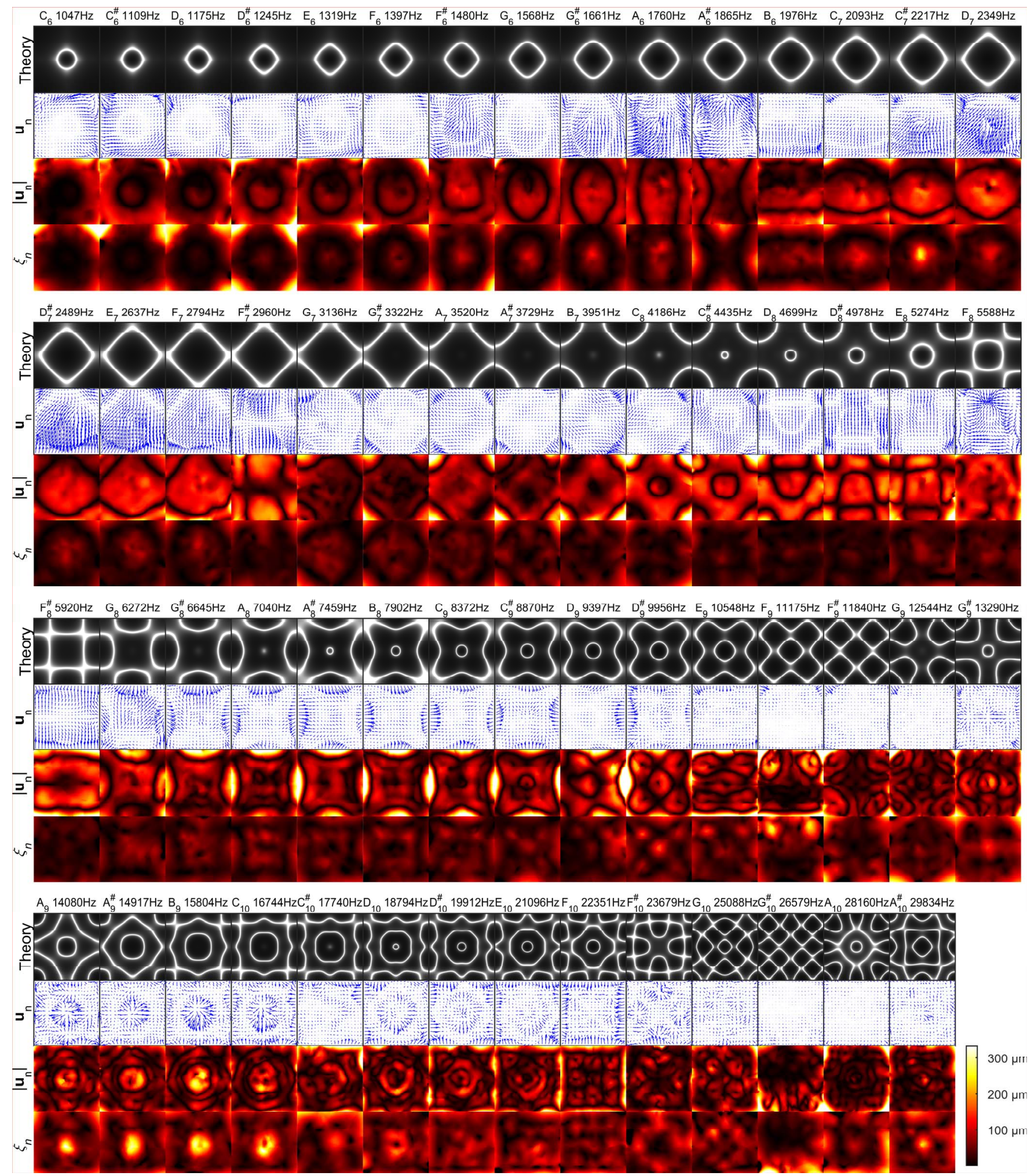

Fig. 3. Comparison of theoretical Chladni figures and statistically modeled displacement fields for the notes. First row shows the theoretically computed Chladni figure, second row shows the modelled vector field, third row shows the absolute displacement, and the fourth row shows the residual. Adapted from [24]. 
from the given position. In the implementation, we represent a single particle with multiple neighbor points that spread around the given position at certain radius. The radius is chosen to represent the maximum initial position error. The note for each step is then selected such that the displacement vectors will produce net motions of all neighbor points toward the target. Thus we call this method as Neighborhood Net Motion (NNM).

\section{B. Closed-loop control strategy}

We implemented closed-loop control schemes to plan and control the motion of objects on the plate. We use a camera to capture the top-view of the plate, and repeatedly measure the position of the objects on the plate. In every step, we use the model to choose a note that moves the objects towards their desired directions. Two methods have been used for this purpose: (1) model predictive control (MPC) and (2) linear programming.

\section{1) Model predictive control (MPC)}

Our model predictive controller is based on iterative optimization of the nonlinear cost function $J$ for a finite horizon. The system is assumed Markovian where the current state of the system (position of the objects) is independent from the history. At time step $t$, positions of the objects are sampled and the optimization cost function $J$ is calculated for all mode combinations until time horizon $T$. The optimization cost function $J$ is represented as

$$
J_{n}=\sum_{m=1}^{M} \sum_{i=1}^{T} w_{1}\left|r_{m}-E\left(x_{m i}\right)\right|+w_{2} \xi_{n}^{2}\left(E\left(x_{m i}\right)\right)
$$

Where $M$ denotes the number of particles, $i$ is the current time horizon, $w$ is the weighting factor, $r_{m}$ is the target position for particle $m, E\left(x_{m i}\right)$ is the expected position of object $m$ in time horizon $i$, and $\xi_{n}{ }^{2}$ is the residual field calculated for each mode as a function of position. In time horizon 1, we use the measured current location of the object instead of expected position. In every time step, only the first calculated note is played, then the position is sampled again and the calculations are repeated starting from the new current position. We used $T=2$ in our control experiments presented in section V.B.

\section{2) Linear programming}

In each time step, the computer solves a linear programming problem and calculates a linear combination of all the modelled displacement fields such that the net motion for all the objects at their current position is guided towards the desired direction. The new weight of each note is then added to the previously accumulated weight of that note. Then the note with the highest accumulated weight is played and its accumulated weight is reset to zero. The procedure is repeated in the following time steps until the targets are reached. The details of the control method are explained thoroughly in [24].

\section{RESULTS}

All control strategies have been experimentally validated in manipulating particles. The particles are usually from the same batch of particles used for modelling the displacement fields unless explicitly stated.

\section{A. Open-loop control result}

In open-loop control, we replayed the recorded sequence of note from simulation to move the particle on the real plate from its initial position in predefined trajectory without any position feedback. The results are shown in Fig. 4 for various trajectories with the sequence of notes generated from direct method and

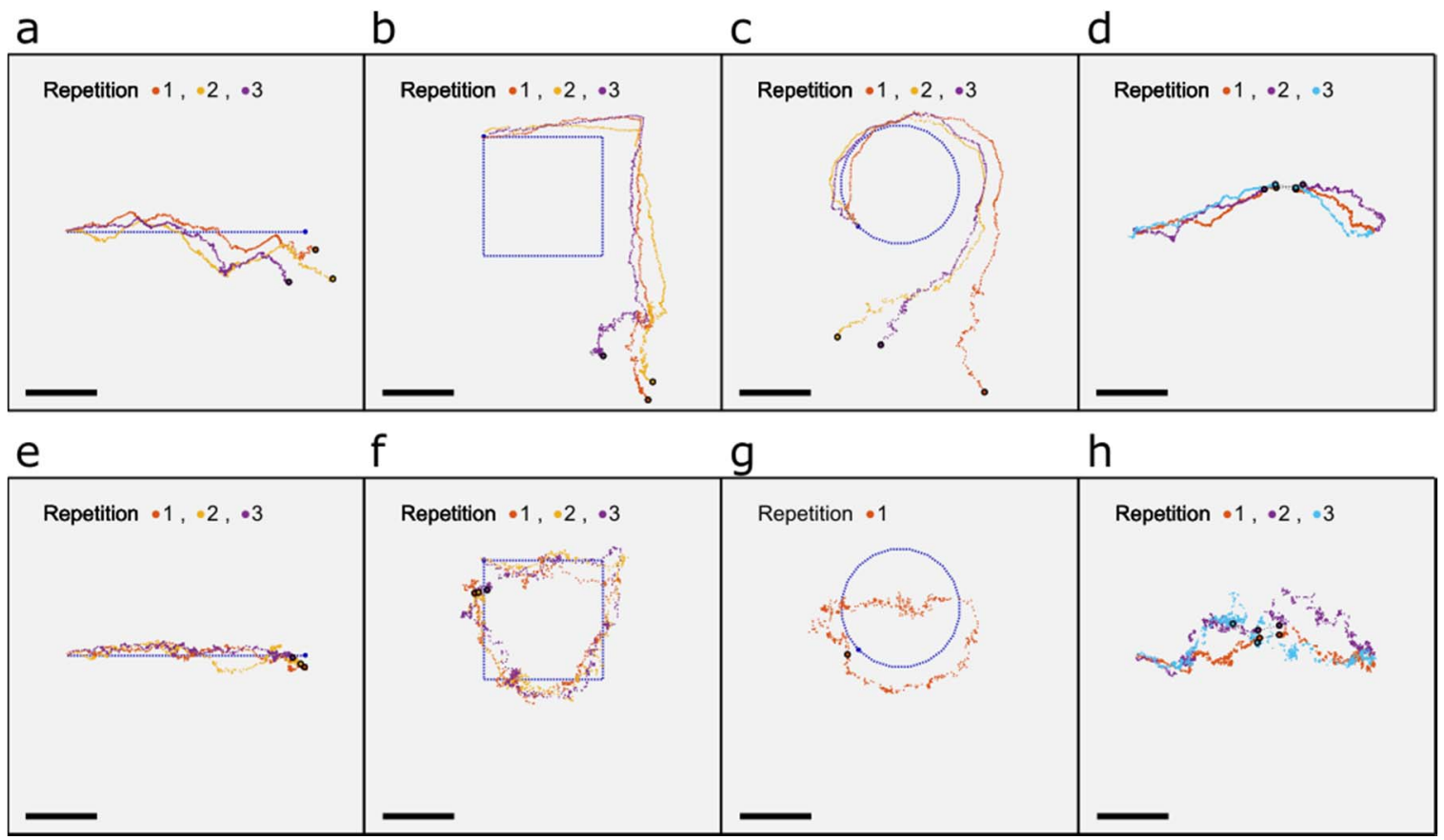

Fig. 4. Particle trajectories of open-loop control on real plate for various pre-defined shape (a-c and e-g) and particles merging (d and h). First row (a-d) are the results when replaying the sequence of notes generated from direct method. Second row (e-h) are the results when replaying the sequence of notes generated from NNM method. Scale bar is $6 \mathrm{~mm}$ for (a, e) and $3 \mathrm{~mm}$ for (b,c,d,f,g,h). 


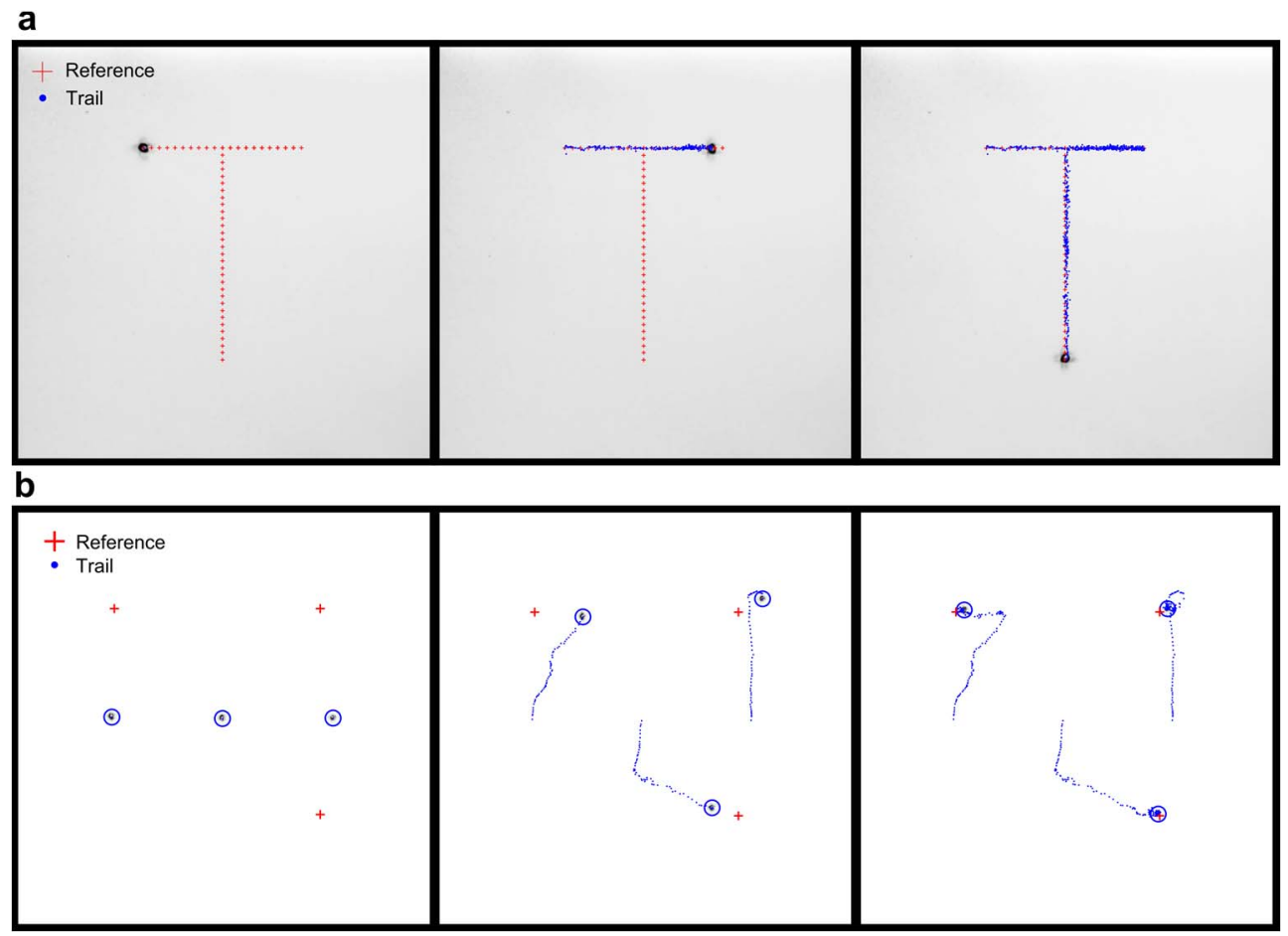

Fig. 5. Manipulation of single and multiple particles using MPC controller. (a) Trajectory following of a $750 \mu \mathrm{m}$ spherical glass bead along a T-shaped path. (b) Simultaneous manipulation of three solder balls using MPC method.

NNM method. By using the direct method, the manipulation task for line, square, and circle trajectories was completed in 1639, 931, and 705 control steps, respectively. However, NNM took considerably more control steps to accomplish the manipulation tasks. To complete the line, square, and circle trajectories, NNM required 6173,7595 , and 10075 control steps, respectively.

We also performed particles merging experiment, in which two separated particles are moved simultaneously to merge by minimizing the distance between the particles. The sequence of notes were generated in simulation with direct and NNM method. The result is also shown in Fig. 4. In average, the final center-to-center distance for the direct method and NNM method were approximately $1.1 \mathrm{~mm}$ and $1.0 \mathrm{~mm}$, respectively, while the diameter of the particles were slightly larger than 600 $\mu \mathrm{m}$. In average, the particle merging was completed by the direct and NNM method in 678 and 1457 control steps, respectively.

\section{B. Closed-loop control result}

We have successfully demonstrated motion control of single and multiple particles on the Chladni plate using both the MPC and the linear programming controllers.

Multiple trajectory following experiments was carried out with single and multiple particles. By using closed-loop MPC method, we manipulated a $750 \mu \mathrm{m}$ spherical glass bead along a T-shaped trajectory (Fig. 5a) in 664 control steps. In another experiment, we performed point-to-point transportation of three solder balls towards predefined targets (Fig. 5b) in 551 control steps. We also demonstrated trajectory following with one (Fig. 6a) and three particles (Fig. 6b) using the closed-loop linear programming controller in 1181 and 1580 control steps, respectively.
Sorting objects into distinguished groups is a widely applicable task. In (Fig. 6c), the linear programming controller was used for sorting of six particles into two groups. The particles were initially placed in arbitrary positions on the plate. Then, the controller manipulated three of the particles towards the upper right corner of the plate and the others towards the lower left corner of the plate in 11030 control steps. At the end of the experiment, the particles were well-divided into two distinct groups. Furthermore, we performed pattern transformation utilizing the linear programming controller. Four different types of objects, a mustard seed, a chia seed, a candy ball, and a surface mount technology (SMT) resistor were placed at their corresponding initial position. The controller then transformed these particles from the initial diamond shape into a square shape (Fig. 6d) in 2173 control steps.

We have also created patterns on the plate using the linear programming controller. In these experiments, we define a target pattern consisting of line segments and select the desired direction of each particle towards the nearest point on a line segment in the pattern. We refer to these patterns as artificial nodal lines, because the particles tend to aggregate along these lines, though the lines do not correspond to any Chladni figures of the plate. We formed a triangular pattern using six solder balls (Fig. 6e) in 2096 control steps. Moreover, we aligned six radio frequency identification (RFID) chips in a line from initially scattered formation (Fig. 6f) in 1375 control steps.

In another application, we loaded $8 \mu 1$ water droplets on two SMT resistors. The resistors were used as the end-effectors of an untethered microrobotic system. We transported the water droplets and merged them into a single droplet (Fig. 7) in 318 control steps. 


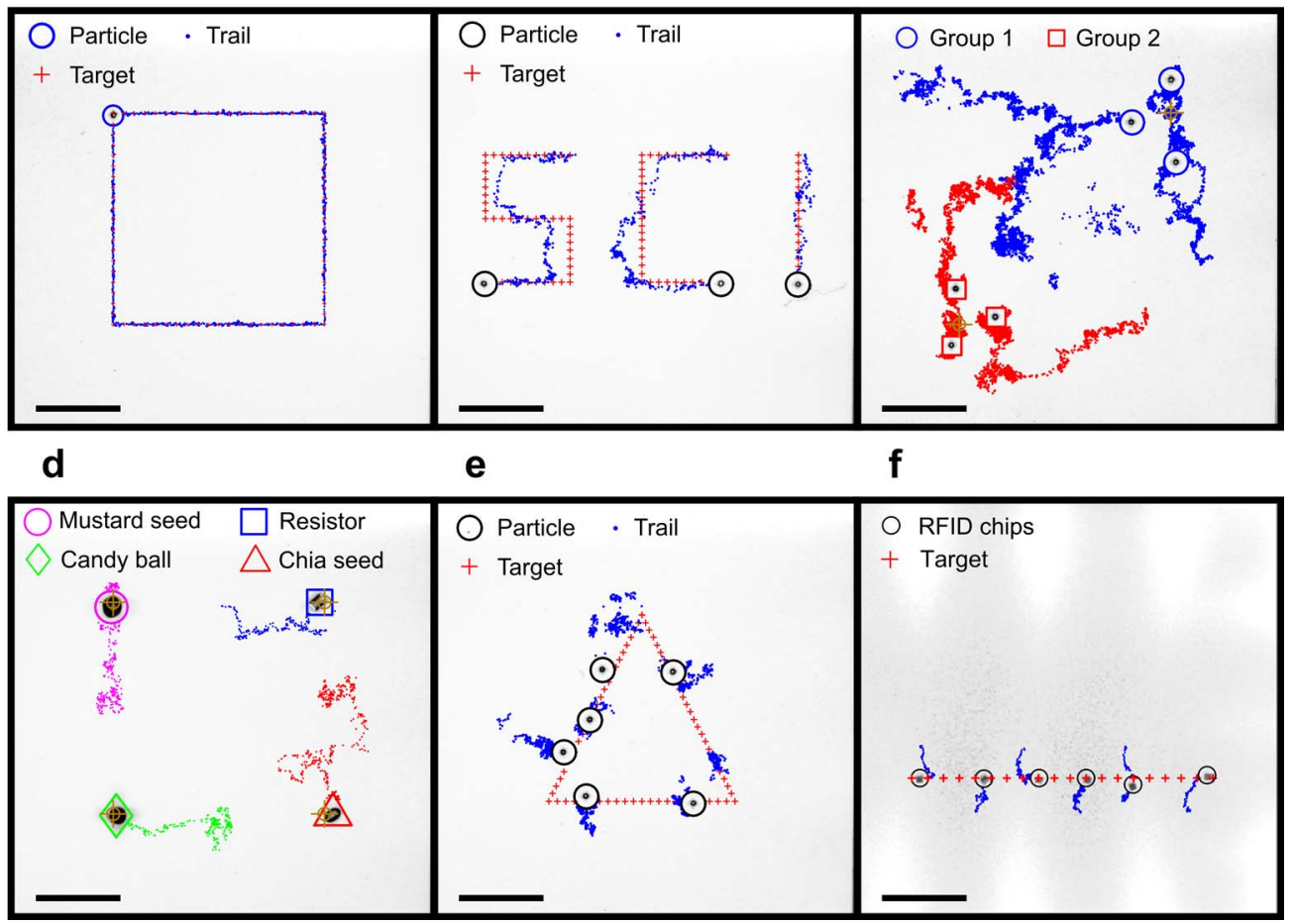

Fig. 6. Manipulation of single and multiple particles using linear programming closed-loop control. (a) Trajectory following of a solder ball along a square-shaped path. (b) Simultaneous manipulation of three solder balls. (c) Particle sorting. (d) Pattern transformation. (e) Formation of a triangular pattern with six solder balls. (f) Aligning six RFIDs on a line. (Scale bar, $1 \mathrm{~cm}),(\mathrm{a}-\mathrm{d}, \mathrm{f})$ are adapted from [24].

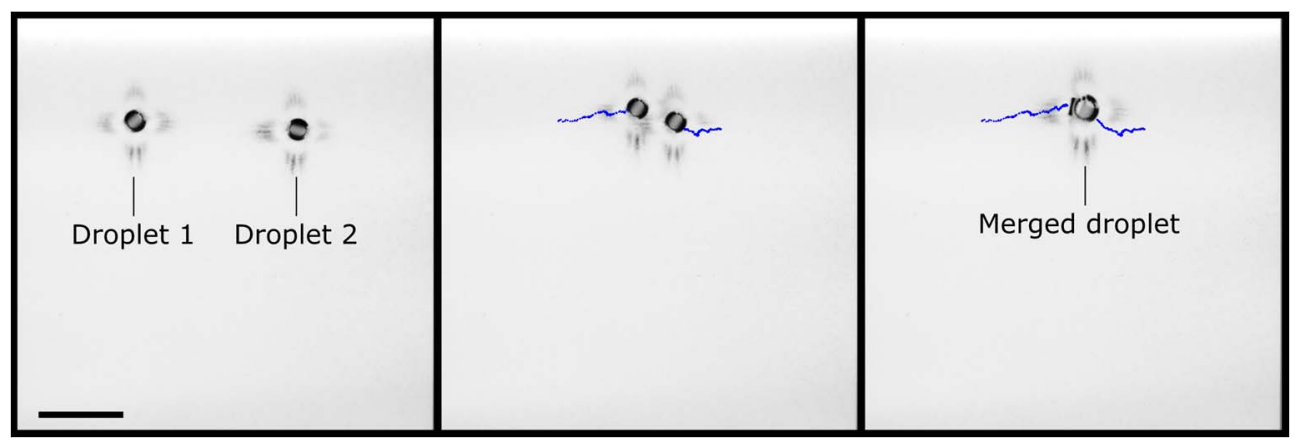

Fig. 7. Untethered acoustic microrobotic system for droplet merging where two $8 \mathrm{ml}$ water droplets on carriers are brought together and merged into a single droplet. (Scale bar, $1 \mathrm{~cm}$ ) [24].

We quantified the achievable manipulation accuracy of our control methods by performing line following experiments and measuring the tracking error from the reference path. A summary of the results are presented in Table. I. The accuracy of line following was calculated with respect to the reference line while repeatability values correspond to standard deviation across actual trajectories. We used the T-shaped trajectory following experiment (Fig. 5a) to quantify the accuracy of the MPC method. The measured data show an accuracy mean error of $17 \mu \mathrm{m}$ and a standard deviation of $142 \mu \mathrm{m}$. We performed square line following experiment to characterize the tracking accuracy of the linear programming controller. We used a 25 $\mathrm{mm} \times 25 \mathrm{~mm}$ square as the reference trajectory, and the experiments were repeated 6 times. The measured data show an accuracy mean error of $4 \mu \mathrm{m}$ and a standard deviation of 125 $\mu \mathrm{m}$. The resolution of our top-view camera is approximately 60 $\mu \mathrm{m}$ in the object space. Therefore, the tracking error can be mainly attributed to the top-view camera resolution. The accuracy of our system could be further improved by increasing the top view resolution from the current $60 \mu \mathrm{m}$ and reducing the duration of the notes.

\section{TABLE I. MANIPULATION ACCURACY AND PRECISION OF THE OPEN-LOOP CONTROLLERS}

\begin{tabular}{|c|c|c|c|}
\hline \multirow{2}{*}{ Trajectory } & $\begin{array}{c}\text { Performance } \\
\text { measure }\end{array}$ & Direct method & NNM method \\
\hline \multirow{3}{*}{ Line } & Accuracy & $\begin{array}{c}1407 \mu \mathrm{m} \\
( \pm 1060 \mu \mathrm{m})\end{array}$ & $\begin{array}{c}636 \mu \mathrm{m} \\
( \pm 511 \mu \mathrm{m})\end{array}$ \\
\cline { 2 - 4 } & Repeatability & $511 \mu \mathrm{m}$ & $245 \mu \mathrm{m}$ \\
\hline \multirow{3}{*}{ Square } & Accuracy & N.A. & $\begin{array}{c}428 \mu \mathrm{m} \\
( \pm 352 \mu \mathrm{m})\end{array}$ \\
\cline { 2 - 4 } & Repeatability & $314 \mu \mathrm{m}$ & $155 \mu \mathrm{m}$ \\
\hline
\end{tabular}


Total manipulation time is determined by duration of each note and number of control steps. Duration of notes in open-loop and closed-loop experiments were 100 and $500 \mathrm{~ms}$, respectively, except in Fig. 7 where we used 250 ms. Number of manipulation steps is affected by number of particles, manipulation trajectory length, as well as availability of optimal vector fields towards the target locations.

\section{CONCLUSION}

This paper has shown a new route to independent and simultaneous control of multiple objects where the properties of the objects can be rather relaxed. The fundamental idea is to employ spatially highly nonlinear excitation fields allowing independent and simultaneous motion control of particles. The hardware apparatus is extremely simple: a vibrating plate mounted on a single piezoelectric actuator. The displacement of particles on the plate has been modelled using particle tracking velocimetry and the model has been used in various open-loop and closed-loop control methods.

In open-loop control experiments, we replayed the precalculated sequence of notes to move the particle along predefined trajectories. The actual trajectory tends to deviate from the predefined trajectory as number of steps increases. The Neighborhood Net Motion (NNM) method allows trajectory following with reasonable deviations. In closed-loop control experiments, we used vision feedback control. It significantly improves the control accuracy, thus enabling complex manipulation tasks. We implemented model predictive control (MPC) and linear programming methods to accomplish various tasks, including trajectory following, particle sorting, pattern formation, and merging droplets.

Despite the reported method is based on acoustic manipulation on a Chladni plate, the proposed methods can be extended to other energy fields provided that they are spatially highly nonlinear and such nonlinearity can be excited.

\section{ACKNOWLEDGMENT}

This work was supported by Academy of Finland (grant 295006 and grant 296250), LPDP Scholarship of Indonesia, and Aalto doctoral school of Electrical Engineering. H. Wijaya and K. Latifi contributed equally to this work. The authors acknowledge Veikko Sariola and Ville Liimatainen for their help during the course of this work.

\section{REFERENCES}

[1] C. R. P. Courtney, C. E. M. Demore, H. Wu, A. Grinenko, P. D. Wilcox, S. Cochran, and B. W. Drinkwater, "Independent trapping and manipulation of microparticles using dexterous acoustic tweezers," Appl. Phys. Lett., vol. 104, no. 15, p. 154103, 2014.

[2] L. Y. Yeo and J. R. Friend, "Ultrafast microfluidics using surface acoustic waves," Biomicrofluidics, vol. 3, no. 1, pp. 1-23, 2009.

[3] J. Friend and L. Y. Yeo, "Microscale acoustofluidics: Microfluidics driven via acoustics and ultrasonics," Rev. Mod. Phys., vol. 83, no. 2, pp. 647-704, 2011.

[4] X. Ding, S.-C. S. Lin, B. Kiraly, H. Yue, S. Li, I.-K. Chiang, J. Shi, S. J. Benkovic, and T. J. Huang, "On-chip manipulation of single microparticles, cells, and organisms using surface acoustic waves," Proc. Natl. Acad. Sci., vol. 109, no. 28, pp. 11105-11109, 2012.

[5] T. Laurell, F. Petersson, and A. Nilsson, "Chip integrated strategies for acoustic separation and manipulation of cells and particles.," Chem. Soc. Rev., vol. 36, no. 3, pp. 492-506, 2007.
[6] D. J. Collins, B. Morahan, J. Garcia-Bustos, C. Doerig, M. Plebanski, and A. Neild, "Two-dimensional single-cell patterning with one cell per well driven by surface acoustic waves.," Nat. Commun., vol. 6, p. 8686, 2015.

[7] P. Chen, Z. Luo, S. Güven, S. Tasoglu, A. V. Ganesan, A. Weng, and U. Demirci, "Microscale Assembly Directed by Liquid-Based Template," Adv. Mater., vol. 26, no. 34, pp. 5936-5941, 2014.

[8] A. R. Rezk, J. K. Tan, and L. Y. Yeo, "HYbriD Resonant Acoustics (HYDRA)," Adv. Mater., pp. 1970-1975, 2016.

[9] Y. Zhang and T. H. Wang, "Full-range magnetic manipulation of droplets via surface energy traps enables complex bioassays," $A d v$. Mater., vol. 25, no. 21, pp. 2903-2908, 2013.

[10] B. R. Donald, C. G. Levey, and I. Paprotny, "Planar microassembly by parallel actuation of MEMS microrobots," J. Microelectromechanical Syst., vol. 17, no. 4, pp. 789-808, 2008.

[11] R. Fujiwara, P. Hemthavy, K. Takahashi, and S. Saito, "Pulse voltage determination for electrostatic micro manipulation considering surface conductivity and adhesion of glass particle," $A I P A d v$., vol. 5, no. 5, 2015.

[12] D. R. Frutiger, K. Vollmers, B. E. Kratochvil, and B. J. Nelson, "Small, Fast, and under Control: Wireless Resonant Magnetic Micro-agents," Springer Tracts Adv. Robot., vol. 54, no. 2004, pp. 169-178, 2009.

[13] R. S. M. Rikken, R. J. M. Nolte, J. C. Maan, J. C. M. van Hest, D. a Wilson, and P. C. M. Christianen, "Manipulation of micro- and nanostructure motion with magnetic fields.," Soft Matter, vol. 10, no. 9, pp. 1295-308, 2014.

[14] H. Maruyama, T. Fukuda, and F. Arai, "Laser manipulation and optical adhesion control of functional gel-microtool for on-chip cell manipulation," 2009 IEEE/RSJ Int. Conf. Intell. Robot. Syst. IROS 2009, pp. 1413-1418, 2009.

[15] O. J. Sul, M. R. Falvo, R. M. Taylor, S. Washburn, and R. Superfine, "Thermally actuated untethered impact-driven locomotive microdevices," Appl. Phys. Lett., vol. 89, no. 20, pp. 1-4, 2006.

[16] A. A. Solovev, Y. Mei, E. B. Ureña, G. Huang, and O. G. Schmidt, "Catalytic microtubular jet engines self-propelled by accumulated gas bubbles," Small, vol. 5, no. 14, pp. 1688-1692, 2009.

[17] S. Martel, M. Mohammadi, O. Felfoul, Zhao Lu, and P. Pouponneau, "Flagellated Magnetotactic Bacteria as Controlled MRI-trackable Propulsion and Steering Systems for Medical Nanorobots Operating in the Human Microvasculature," Int. J. Rob. Res., vol. 28, no. 4, pp. 571$582,2009$.

[18] E. D. Diller, S. Floyd, C. Pawashe, and M. Sitti, "Control of multiple heterogeneous magnetic micro-robots on non-specialized surfaces," Proc. - IEEE Int. Conf. Robot. Autom., vol. 28, no. 1, pp. 115-120, 2011.

[19] E. Diller, J. Giltinan, P. Jena, and M. Sitti, "Three dimensional independent control of multiple magnetic microrobots," Proc. - IEEE Int. Conf. Robot. Autom., pp. 2576-2581, 2013.

[20] J. deSa, Q. Zhang, E. Ergezen, and R. Lec, "Microparticle manipulation on the surface of a piezoceramic actuator - art. no. 105803," Meas Sci Technol, vol. 21, no. 10, p. 5803, 2010.

[21] D. S. Reznik, J. F. Canny, and N. Alldrin, "Leaving on a plane jet," Proc. 2001 IEEE/RSJ Int. Conf. Intell. Robot. Syst., vol. 1, pp. 202-207, 2001.

[22] D. S. Reznik and J. F. Canny, "C'mon part, do the local motion!," Proc. 2001 ICRA. IEEE Int. Conf. Robot. Autom. (Cat. No.01CH37164), vol. 3, pp. 2235-2242, 2001.

[23] T. H. Vose, P. Umbanhowar, and K. M. Lynch, "Friction-Induced Velocity Fields for Point Parts Sliding on a Rigid Oscillated Plate," Int. J. Rob. Res., vol. 28, no. 8, pp. 1020-1039, 2009.

[24] Q. Zhou, V. Sariola, K. Latifi, and V. Liimatainen, "Controlling the motion of multiple objects on a Chladni plate," Nat. Commun., pp. 1-10, 2016.

[25] M. J. Gander and F. Kwok, "Chladni Figures and the Tacoma Bridge: Motivating PDE Eigenvalue Problems via Vibrating Plates," SIAM Rev., vol. 54, no. 3, pp. 573-596, 2012.

[26] P. H. Tuan, C. P. Wen, P. Y. Chiang, Y. T. Yu, H. C. Liang, K. F. Huang, and Y. F. Chen, "Exploring the resonant vibration of thin plates: Reconstruction of Chladni patterns and determination of resonant wave numbers," J. Acoust. Soc. Am., vol. 137, no. 4, pp. 2113-2123, 2015. 